\title{
EQUITABLE COLORING AND EQUITABLE CHOOSABILITY OF GRAPHS WITH SMALL MAXIMUM AVERAGE DEGREE ${ }^{1}$
}

\author{
Aijun Dong \\ School of Science \\ Shandong Jiaotong University \\ Jinan, 250023, P.R. China \\ e-mail: dongaijun@mail.sdu.edu.cn
}

AND

Xin Zhang

School of Mathematics and Statistics

Xidian University

Xi'an, 7100'1, P.R. China

e-mail: xzhang@xidian.edu.cn

\begin{abstract}
A graph is said to be equitably $k$-colorable if the vertex set $V(G)$ can be partitioned into $k$ independent subsets $V_{1}, V_{2}, \ldots, V_{k}$ such that ||$V_{i}|-| V_{j}|| \leq$ $1(1 \leq i, j \leq k)$. A graph $G$ is equitably $k$-choosable if, for any given $k$-uniform list assignment $L, G$ is $L$-colorable and each color appears on at most $\left[\frac{|V(G)|}{k}\right\rceil$ vertices. In this paper, we prove that if $G$ is a graph such that $\operatorname{mad}(G)<3$, then $G$ is equitably $k$-colorable and equitably $k$ choosable where $k \geq \max \{\Delta(G), 4\}$. Moreover, if $G$ is a graph such that $\operatorname{mad}(G)<\frac{12}{5}$, then $G$ is equitably $k$-colorable and equitably $k$-choosable where $k \geq \max \{\Delta(G), 3\}$.
\end{abstract}

Keywords: graph coloring, equitable choosability, maximum average degree.

2010 Mathematics Subject Classification: 05C15.

\footnotetext{
${ }^{1}$ This work was supported by the National Natural Science Foundation of China (Grant No. 71571111). It was also supported by China Postdoctoral Science Foundation Funded Project (Grant No.2014M561909); the Nature Science Foundation of Shandong Province of China (Grant No. ZR2014AM028; ZR2014GL001; ZR2014FM033), the Natural Science Basic Research Plan in Shaanxi Province of China (No. 2017JM1010) and the Fundamental Research Funds for the Central Universities (No. JB170706).
} 


\section{REFERENCES}

[1] J.A. Bondy and U.S.R. Murty, Graph Theory with Applications (North-Holland, New York, 1976).

[2] B.L. Chen, K.W. Lih and P.L. Wu, Equitable coloring and the maximum degree, European J. Combin. 15 (1994) 443-447. doi:10.1006/eujc.1994.1047

[3] B.L. Chen and K.W. Lih, Equitable coloring of trees, J. Combin. Theory Ser. B 611 (1994) 83-87. doi:10.1006/jctb.1994.1032

[4] B.L. Chen and C.H. Yen, Equitable $\Delta$-coloring of graphs, Discrete Math. 312 (2012) 1512-1517. doi:10.1016/j.disc.2011.05.020

[5] A.J. Dong, X. Tan, X. Zhang and G.J. Li, Equitable coloring and equitable choosability of planar graphs without 6- and 7-cycles, Ars Combin. 103 (2012) 333-352.

[6] A.J. Dong, X. Zhang and G.J. Li, Equitable coloring and equitable choosability of planar graphs without 5- and 7-cycles, Bull. Malays. Math. Sci. Soc. 35 (2012) 897-910.

[7] A.J. Dong, G.J. Li and G.H. Wang, Equitable and list equitable colorings of planar graphs without 4-cycles, Discrete Math. 313 (2013) 1610-1619. doi:10.1016/j.disc.2013.04.011

[8] A.J. Dong, Q.S. Zou and G.J. Li, Equitable and list equitable colorings of graphs with bounded maximum average degree, Ars Combin. 124 (2016) 303-311.

[9] A. Hajnal and E. Szemerédi, Proof of a conjecture of Erdős, in: A. Rényi, V.T. Sós (Eds.), Combinatorial Theory and Its Applications (North-Holland, Amsterdam, 1970) 601-623.

[10] H.A. Kierstead and A.V. Kostochka, Equitable list coloring of graphs with bounded degree, J. Graph Theory 74 (2013) 309-334. doi:10.1002/jgt.21710

[11] A.V. Kostochka, M.J. Pelsmajer and D.B. West, A list analogue of equitable coloring, J. Graph Theory 47 (2003) 166-177. doi:10.1002/jgt.10137

[12] A.V. Kostochka and K. Nakprasit, Equitable colorings of $k$-degenerate graphs, Combin. Probab. Comput. 12 (2003) 53-60. doi: $10.1017 / \mathrm{S} 0963548302005485$

[13] A.V. Kostochka and K. Nakprasit, Equitable $\Delta$-colorings of graphs with low average degree, Theoret. Comput. Sci. 349 (2005) 82-91. doi:10.1016/j.tcs.2005.09.031

[14] K.W. Lih and P.L. Wu, On equitable coloring of bipartite graphs, Discrete Math. 151 (1996) 155-160. doi:10.1016/0012-365X(94)00092-W 
[15] K.W. Lih, Equitable Coloring of Graphs (Springer Science+Business Media, New York, 2013).

[16] Q. Li and Y.H. Bu, Equitable list coloring of planar graphs without 4- and 6-cycles, Discrete Math. 309 (2009) 280-287.

doi:10.1016/j.disc.2007.12.070

[17] R. Luo, J.S. Sereni, D.C. Stephens and G. Yu, Equitable coloring of sparse planar graphs, SIAM J. Discrete Math. 24 (2010) 1572-1583. doi:10.1137/090751803

[18] W. Meyer, Equitable coloring, Amer. Math. Monthly 80 (1973) 920-922. doi:10.2307/2319405

[19] K. Nakprasit, Equitable colorings of planar graphs with maximum degree at least nine, Discrete Math. 312 (2012) 1019-1024. doi:10.1016/j.disc.2011.11.004

[20] K. Nakprasit and K. Nakprasit, Equitable colorings of planar graphs without short cycles, Theoret. Comput. Sci. 465 (2012) 21-27. doi:10.1016/j.tcs.2012.09.014

[21] M.F. Pelsmajer, Equitable list coloring for graphs of maximum degree 3, J. Graph Theory 47 (2004) 1-8. doi:10.1002/jgt.20011

[22] W.F. Wang and K.W. Lih, Equitable list coloring of graphs, Taiwanese J. Math. 8 (2004) 747-759. doi:10.11650/twjm/1500407716

[23] W.F. Wang and K.M. Zhang, Equitable colorings of line graphs and complete rpartite graphs, System Sci. Math. Sci. 13 (2000) 190-194.

[24] J.L. Wu and P. Wang, Equtiable coloring planar graphs with large girth, Discrete Math. 308 (2008) 985-990. doi:10.1016/j.disc.2007.08.059

[25] Z. Yan and W. Wang, Equitable coloring of Kronecker products of complete multipartite graphs and complete graphs, Discrete Appl. Math. 162 (2014) 328-333. doi:10.1016/j.dam.2013.08.042

[26] H.P. Yap and Y. Zhang, The equitable $\Delta$-coloring conjecture holds for outerplanar graphs, Bull. Inst. Math. Acad. Sin. 25 (1997) 143-149.

[27] H.-P. Yap and Y. Zhang, Equitable colorings of planar graphs, J. Combin. Math. Combin. Comput. 27 (1998) 97-105.

[28] X. Zhang and J.L. Wu, On equitable and equitable list coloring of series-parallel graphs, Discrete Math. 311 (2011) 800-803. doi:10.1016/j.disc.2011.02.001

[29] J.L. Zhu and Y.H. Bu, Equitable list colorings of planar graphs without short cycles, Theoret. Comput. Sci. 407 (2008) 21-28. doi:10.1016/j.tcs.2008.04.018 
[30] J.L. Zhu, Y.H. Bu and X. Min, Equitable list-coloring for $C_{5}$-free plane graphs without adjacent triangles, Graphs Combin. 31 (2015) 795-804. doi:10.1007/s00373-013-1396-7

[31] J.L. Zhu and Y.H. Bu, Equitable and equitable list colorings of graphs, Theoret. Comput. Sci. 411 (2010) 3873-3876.

doi:10.1016/j.tcs.2010.06.027

Received 10 October 2016

Revised 16 February 2017

Accepted 16 February 2017 